Unter Dermatologen führt die aggressive Kampagne der FMH zur Förderung von Managed Care (MC) schweizweit zu einem nicht mehr überhörbaren Unmut. Dermatologen beschäftigen sich in erster Linie mit der Oberfläche ihrer Kundschaft. Situationsgegeben sind Fehler in Diagnostik und Therapie leichter erkennbar als in anderen medizinischen Spezialitäten. Die Aussage, dass MC einer Verbesserung der medizinischen Versorgungsqualität gleichkomme, muss aus Sicht der Dermatologen klar in Abrede gestellt werden. Die hier vorgestellte Publikation hat die offizielle Unterstützung der SGDV wie auch der FMCH.

\title{
Ohne Hausarztmedizin funktioniert unser Gesundheitssystem nicht, ohne Hausarztmodell jedoch bestens!
}

\author{
oder: Die Hausarztmedizin soll sich durch ihre Qualität und nicht durch Zwang \\ dem Kunden aufdrängen!
}

Thomas Hofer

Korrespondenz:

Dr. med. Thomas Hofer Winkelriedstrasse 10 CH-5430 Wettingen

thomas.hofer@active.ch
Unser Gesundheitssystem geniesst grosse Aufmerksamkeit in Medien, Politik und Bevölkerung. Anlass dazu geben dessen enorme Kostenzunahme in den vergangenen Jahrzehnten und die Ungewissheit über seine zukünftige Kostenentwicklung. Eben hat die Diskussion zum Thema Einheitskrankenkasse uns Schweizer - gedanklich mehr als wörtlich - mobilisiert. In ihr wurde, sowohl von einzelnen Parteien wie auch von in der Grundversorgung tätigen Ärzteverbänden, ein Lösungsansatz zur Problematik der Kostenexplosion gesehen. Die Einheitskasse wurde vom Volk deutlich abgelehnt. Schon wird mit Nachdruck der nächste Lösungsvorschlag als unabdingbare Voraussetzung für die Sicherung unseres Gesundheitssystems gefordert: Managed Care (MC) soll es richten!

Seit etwa 20 Jahren «werkelt» man in der Schweiz an neuen Versicherungsmodellen wie Hausarzt-, HMO- oder Bonussystem herum. Neu im wörtlichen Sinne sind diese Modelle nicht, gibt es sie doch schon seit Jahrzehnten in diversen Ländern unserer Erde. Die Begeisterung in der Schweiz für solche Modelle hielt und hält sich aber in Grenzen. Lediglich 10\% der Bevölkerung haben sich 2006 für ein solches alternatives Versicherungsmodell entschieden. Die Gründe für dieses Desinteresse sind (obwohl wahrscheinlich bekannt) nicht publiziert. Seit ein paar Monaten werden diese Systeme aber erneut äusserst aktiv dem Gesunden und Kranken angepriesen. Auf Ärzteseite sind es in erster Linie die in der Grundversorgung Engagierten, die vom Bund diesbezüglich Schützenhilfe verlangen.
Entsprechende Motivationspapiere wurden den eidgenössischen Räten verteilt [1]. Auf Versichererseite wird MC ebenfalls favorisiert, allerdings mit der Einschränkung, dass nur die (aus welchen Gründen auch immer) ihnen genehmen Hausärzte als Leistungserbringer vertraglich in dieses Modell eingebunden sind. Trotz dieser auf den ersten Blick gleichen Zielvorgabe könnte die Motivation dieser beiden Gruppierungen nicht unterschiedlicher sein: Während die ärztlichen Propagandisten durch diese Aktivität der drohenden Aufhebung des Vertragszwanges mit den Krankenkassen entgegenwirken wollen, bezwekken die Krankenkassen mit ihrem Vorgehen eben gerade das Gegenteil (Stichwort: Aufhebung des Kontrahierungszwanges). Die Grundversorger wollen zudem mit ihrem Vorgehen der Bedeutung der Hausarztmedizin mehr Nachdruck verleihen.

Angesichts dieses berufspolitisch (Ärzte) bzw. geschäftspolitisch (Krankenkassen) geprägten Hintergrundes erhält die heutige Forderung nach mehr MC eine Gewichtung, die ihr nicht gebührt und die dazu verleitet, Fragliches, gar Problematisches, das dem MC innewohnt, nicht mehr mit der gebührenden Objektivität zu durchleuchten. Politisches dominiert Sachliches. So propagiert die Ärzteseite ganz selbstverständlich, dass «MC bekannterweise mit einer Qualitätsverbesserung verbunden» sei. Dies, obwohl bis heute keine standardisierten Kriterien existieren, welche die Qualität der ärztlich erbrachten Leistung überhaupt definieren würden. Wenn das Gesundheitssystem Dänemarks, das sich 
durch Einheitskasse und striktes MC definiert, im Vergleich zu unserem Gesundheitswesen von an MC interessierten Schweizer Ärzten [2] fast etwas wehmütig als Wunscharbeitsfeld vorgestellt wird («we are impressed and a bit depressed») und dabei die um drei (!) Jahre kürzere Lebenserwartung der Dänen gegenüber derjenigen der restlichen Europäer lediglich durch ihre «ungesunde und weniger schmackhafte Ernährung», die dann eben auch vermehrt Herzgefässerkrankungen zur Folge habe, erklärt wird, so hat sich kritisches Denken diesem System gegenüber nicht durchsetzen können. Die durchschnittliche Lebenserwartung eines Volkes ist nämlich nicht lediglich abhängig zu machen von den Essgewohnheiten der Bevölkerung. Sondern alle Beteiligten, sowohl die Bevölkerung wie auch die Leistungserbringer (Ärzte, anderes Medizinalpersonal) und die Kostenträger (Krankenkassen und Staat), sind dafür verantwortlich. Im Beispiel Dänemark darf man somit nicht nur den sich ungesund und wenig schmackhaft ernährenden Dänen die Schuld (!) zuschieben, dass sie früher sterben, sondern auch das System Einheitskasse wie auch das strikte MC müssen sich unangenehme Fragen zur verkürzten Lebenserwartung ihrer Bevölkerung gefallen lassen.

«Wenn du nicht willst, so brauch' ich Gewalt!» So könnte man etwas pointiert umschreiben, mit welchen neuen Massnahmen heute MC-interessierte Ärzte dieses Modell in der Schweiz fördern wollen: Wer sich nicht in MC einbinden lässt, soll neu mit einem doppelt so hohen Selbstbehalt behaftet werden wie derjenige, der mitmacht. Und wer mitmacht, soll sich nicht schon nach einem Jahr, sondern neu erst nach drei Jahren wieder aus MC herauslösen können. Finden sich etwa versteckt in diesen neuen Vorschlägen die Antworten auf die Frage, warum sich MC bis heute in der Schweiz nicht durchsetzen konnte? Wurde die «mit MC bekannterweise verbundene Qualitätsverbesserung» von den bis jetzt MC Wählenden nicht erkannt, oder vermissten sie jeweils schon nach kurzer Zeit Leistungen oder gar Qualität, die sie dann ausserhalb MC bekamen? Müsste man sich somit nicht eher Fragen zur Qualität des heute angebotenen MC stellen?

Nach wie vor sind nur etwa $12 \%$ der Bevölkerung bereit, vorbehaltlos auf die freie Arztwahl zu verzichten. Mit etwas Zwang könnte man nun dieser Bereitschaft nachhelfen, vor allem unter den ärmeren Bevölkerungsschichten, die sich ja ohnehin keine weitere Prämienvergünstigung durch einen höheren Selbstbehalt leisten können. Denn die Entscheidung, beispielsweise dreitausend Franken Selbstbehalt zur Not eben aus dem eigenen Sack zu bezahlen, kann nur derjenige treffen, der überhaupt dreitausend Franken jederzeit im Sack hat!

Konstatiert man, wie das die MC-Befürworter tun, dass MC qualitativ bessere Medizin leiste, so wird damit gleichzeitig konstatiert, dass NichtMC höchstens gute, aber sicher immer schlechtere Medizin leiste als MC. Es kann ja nicht zwei bessere Systeme geben, von denen jeweils das eine immer besser ist als das andere. Die Förderung von MC durch daran Interessierte fördert somit - vielleicht unfreiwillig - die Zweiklassenmedizin, nämlich ein Nebeneinander eines besseren und eines schlechteren Systems (selbstverständlich wird der Kunde schlussendlich entscheiden über besser oder nicht besser!). Ist das unser Ziel? Zwei Pressestimmen, welche die Wahrnehmung betroffener Patienten in Ländern mit ausgebautem MC wiedergeben, sollen zitiert sein: In der Sonntagszeitung vom 19. November 2006 schrieb Frau Elsbeth Gugger, Hollandspezialistin von Schweizer Radio DRS, unter dem Titel «Vom Musterschüler zum Sorgenkind» zum Thema MC: «Wer zu einem Spezialisten möchte, braucht eine Überweisung vom Hausarzt. Diese sind aber überfordert und oft zu stolz, um einen Patienten zum Facharzt zu schicken. Wer eine Überweisung ergattern konnte, wird mit ellenlangen Wartezeiten konfrontiert.» Oder Armin Müller, stellvertretender Chefredaktor der Sonntagszeitung, in der Ausgabe vom 11. Februar 2007, kurz und bündig: «Kein Schweizer würde mit den Dänen tauschen.» Soviel zur Qualität von MC im Ausland. Selbstverständlich, so glauben die Befürworter des heute von neuem forcierten MC, werden wir unser schweizerisches MC besser gestalten können, als alle anderen es vor uns versucht haben! Eine Frage sei dennoch erlaubt: Gibt es in MC Qualitätskriterien, welche die Folgen falscher Behandlungen oder zu später Überweisung zum Spezialisten zu erkennen vermögen, eventuell gar messen lassen?

Im Verlaufe des vergangenen Jahrhunderts hat sich in der Schweiz die Lebenserwartung verdoppelt. Diese Entwicklung begann bereits, bevor der Pillenknick zum Geburtenrückgang geführt hat. Die daraus folgende Überalterung unserer Bevölkerung belastet nun v. a. die Kosten für stationäre Behandlung und Pflegeleistungen. Manfred Manser [3], CEO der grössten Krankenkasse Helsana, hält denn folgerichtig auch fest, dass 2006 die grösste Preissteigerung mit 9\% in den Pflegeheimen und bei der Spitex angefallen sei sowie $2 \%$ bei den stationären Spitalaufenthalten. Die Kosten im ambulanten Bereich hingegen - und hier soll MC zur Lösung der Kostenexplosion ganz speziell beitragen! - waren sogar 
rückläufig (Kommentar überflüssig). Soll man somit die freie Arztwahl ausgerechnet dort opfern, wo am wenigsten Kosten anfallen und wo die Kostensteigerung bereits heute negativ ausgefallen ist? Die Schweiz ist eine der ältesten Demokratien, und kein Volk kann so differenziert und freiwillig zu so vielen Sachgeschäften, sei es in der Familie, in der Gemeinde, auf Kantonsebene oder auf Bundesebene, frei entscheiden. Und hier will man nun - mit etwas finanziellem Zwang - ärmere Bevölkerungsschichten um die Freiheit bringen, den Arzt ihrer Wahl jederzeit neu zu bestimmen, indem man sie dazu verpflichtet, die medizinische Qualität dort zu suchen, wo sie müssen, und nicht dort zu finden, wo sie wollen?

Wir werden in den kommenden zwei bis drei Jahrzehnten, bedingt durch unsere «Altenlast», mit jährlichen Kostensteigerungen in der Pflege und in der stationären Behandlung rechnen müssen. Diese uns heute finanziell belastende Generation hat die Grundlage zu unserem heutigen Reichtum und Sozialstaat geschaffen. Kon- zentrieren wir uns auf eine würdige und schlussendlich dankbare Lösung dieser Aufgabe! Die Förderung von MC hingegen ist ein Nebenschauplatz, der von der eigentlichen Problematik ablenkt und von kurzfristigen Partikularinteressen geprägt ist. Zudem geht MC nicht ohne Leistungsabbau einher. Denn vergessen wir nicht: Nicht alles, was heute für mindestens drei Jahre oder länger auf die lange Bank geschoben wird, damit es sich einsparen lässt, hat sich morgen von selbst oder ohne Qualitätseinbusse für die Betroffenen erledigt.

Und zum Schluss noch die spontane Meinung einer Person, die MC im Ausland erfahren hat: «Managed care? Me? I prefer careful managing!»

\section{Literatur}

1 Thesen der FMH zu Managed Care vom 4. Dezember 2006.

2 Zürcher H, Metzger K. Positive Eindrücke vom dänischen Gesundheitswesen. Schweiz Ärztezeitung. 2007;88(3):106-8.

3 MittellandZeitung vom 1. Februar 2007.

\section{Anmerkungen aus der Sicht des Zentralvorstandes der FMH}

Seit der Einführung des KVG vor gut zehn Jahren haben wir Ärztinnen und Ärzte die Möglichkeit, uns in Netzwerken zu organisieren, Verträge mit den Versicherern, Spielregeln zur Qualitätsentwicklung, allenfalls Budgetmitverantwortung auszuhandeln und abzuschliessen. Die Akzeptanz der Modelle ist unterschiedlich, je nach Kanton sind bis zu $30 \%$ der Bevölkerung in solchen Modellen versichert. Im Thesenpapier wird die Rolle des «Caremanagers» beschrieben: Dieser wird in der Regel ein Grundversorger sein, seine Rolle kann aber auch von einem Spezialisten wahrgenommen werden.

Auch wir sind der Ansicht, dass Managed Care mit wissenschaftlicher Begleitung (und nicht aufgrund behaupteter Unterschiede) gefördert wird. Gerade deshalb wird zurzeit viel in Forschung zu Managed Care investiert (vgl. Schweizerisches Gesundheitsobservatorium. Evidenz für Managed Care. Arbeitsdokument 16. 2006).

Die Ärztekammer hat den Zentralvorstand zusätzlich beauftragt, den Know-how-Transfer innerhalb der Ärzteschaft zu unterstützen, das Gespräch zu fördern. Eine gute Gelegenheit, sich vom aktuellen Stand der Entwicklung ein Bild zu machen, aber auch kritische Fragen zu stellen, ist die Teilnahme am Kongress des Forum Managed Care Anfang Juni 2007. Als Zentralvorstand der FMH sind wir gerne bereit, das Gespräch auch im Rahmen einer Vorstandssitzung oder Jahresversammlung einer unserer Gesellschaften fortzusetzen.

Dr. med. Olivier Kappeler, Mitglied des Zentralvorstands der FMH 